\title{
A Fast Reduced Kernel Extreme Learning Machine
}

\author{
Wan-Yu Deng ${ }^{\mathrm{a}, \mathrm{b}}$, Yew-Soon Ong ${ }^{\mathrm{a}, *}$, Qing-Hua Zheng ${ }^{\mathrm{c}}$ \\ ${ }^{a}$ School of Computer Engineering, Nanyang Technological University, Singapore \\ ${ }^{b}$ School of Computer, Xian University of Posts \& Telecommunications, Shaanxi, China \\ ${ }^{c}$ Department of Computer Science and Technology, Xi'an Jiaotong University, China
}

\begin{abstract}
In this paper, we present a fast and accurate kernel-based supervised algorithm referred to as the Reduced Kernel Extreme Learning Machine (RKELM). In contrast to the work in Support Vector Machine (SVM)/Least Square SVM (LS-SVM), which identifies the supports vectors or weights vectors iteratively, the proposed RKELM randomly selects a subset of the available data as support vectors (or mapping samples). By avoiding the iterative steps of SVM, significant cost savings in the training process can be readily attained, especially on Big datasets. RKELM is established based on the rigorous proof of universal learning involving reduced kernel-based SLFN. In particular, we prove that RKELM can approximate any nonlinear functions accurately under the condition of support vectors sufficiency. Experimental results on a wide variety of real world small instance size and large instance size applications in the context of binary classification, multi-class classification, and regression are then reported to show that RKELM can perform at competitive level of generalized performance as the SVM/LSSVM at only a fraction of the computational effort incurred.
\end{abstract}

Keywords: Extreme learning machine; kernel method; support vector machine; RBF network.

\footnotetext{
${ }^{*}$ Corresponding author

Email address: ASYSOng@ ntu .edu.sg (Yew-Soon Ong)
} 


\section{Introduction}

Kernel based learning methods have been extensively used for solving classification and regression problem due to their excellent generalization performance and the mathematical rigor of the field [1]. To date, a plethora of kernel based learning methods such as support vector machine (SVM) [2] and its variants such as LS-SVM [3], RSVM [4] and LLSVM [5] have been proposed for data analysis . In SVM, the training data is first mapped into a higher dimensional feature space through a nonlinear feature mapping function, and the standard optimization method is then used to find the solution of maximizing the separating margin of two different classes in this feature space while minimizing the training errors. Suykens and Vandewalle [2] proposed a least square version of the SVM classifier. Instead of the inequality constraint adopted in the classical SVM, equality constraints are used in the LS-SVM [3]. Hence, by solving a set of linear equations instead of quadratic programming, one can implement the least square approach with ease. LS-SVM is shown to possess excellent generalization performance and low computational cost in many applications. Recently, an unified learning framework for regression and classification termed as Kernel Extreme Learning Machine(KELM) was proposed based on the Extreme Learning Machine (ELM) learning theory and the classification capability theorem. With this universal approximation capability, the bias $b$ in the optimization constraints of SVM, LS-SVM, and PSVM can be removed, and the resultant learning algorith$\mathrm{m}$ has milder optimization constraints. Thus, better generalization performance and lower computational complexity can be obtained. However, on large-scale problems, conventional SVM, LS-SVM and KELM do not scale well in general

In the past decades, many large-scale learning algorithms have been proposed. Lee et al. [4] proposed the reduced support vector machines (RSVM) which restricts the number of candidate support vectors. The main characteristic of this 
method is to reduce the kernel matrix from $N \times N$ to $N \times \tilde{N}$, where $N$ is the number of training instances and $\tilde{N}$ is the size of a randomly selected subset of training data considered as candidates of support vectors. Wang et al. [6] proposed a budgeted stochastic gradient descent approach for training SVMs (BSGD-SVM). The approach keeps the number of support vectors bounded during training through several budget maintenance strategies including removal, projection and merging. Chang etc. [7] proposed a method that uses decision tree to decompose the given data space and train SVMs on the decomposed regions. Results reported indicated notable speed up in training time at competitive test accuracy. LASVM [8], on the other hand, is a one-pass online SVM that involves iterations of sequential minimal optimization (SMO) during each model update so as to remove data samples that are deemed as unlikely to serve as suitable SVs from the training set. Tsang [9] scales up kernel SVM by reformulating SVMs quadratic programming as a minimum enclosing ball problem and then applies an efficient approximation algorithm to obtain a near-optimal solution. In spite of the extensive efforts in the area to cope with the increasing data instance size and dimensionality more elegantly, existing works have mainly focused on developing effective strategies for identifying the optimal set of support vectors. The computational process of identifying support vectors, however can become very intensive, especially when dealing with large-scale data.

In what follows, the core objectives and contributions of the present work are outlined: 1) we will propose a fast non-iterative kernel machine, which is referred to as the fast Reduced Kernel Extreme Learning Machine (RKELM), based on kernel-based learning and extreme learning machine. The key charateristic of the present work is that support vectors are randomly chosen from the training set as opposed to some sophisticated process which is often compute intensive. 2) We prove that RKELM can approximate any nonlinear functions with zero error only 
if the kernel is strictly positive definite and all training data are chosen as support vectors. 3) We analyze the relation of the present work to other related works, such as KELM, ELM and RSVM, and reveal the effects of hidden nodes size and the regularization parameter on generalization performances.

The rest of the paper is organized as follows: Section II gives a brief overview of the classic ELM [10] and the KELM [10]. Section III presents the mathematical derivation of RKELM. The relation of RKELM to other relevant state-of-the-art algorithms is then discussed in Section IV. The performance evaluation and validation of RKELM is subsequently presented in Section V, using commonly used well established datasets from the UCI repository. Last but not least, the brief conclusions and future works are given in Section VI.

\section{A Brief Review of Extreme Learning Machine and its Kernel Extension}

In this section, an overview of the ELM and its kernel extension [10] is presented. This serves to provide the necessary background for the development of the RKELM in Section III.

\subsection{ELM}

The Extreme Learning Machine (ELM) was proposed as a fast learning method for Single-hidden-Layer Feedforward Neural network (SLFN) in [11] [12], where the hidden layer can be any form of piecewise continuous computational functions including Sigmoid, Radial Basis, trigonometric, threshold, fuzzy inference, fully complex, high-order, ridge polynomial, wavelet, etc [13]. In ELM, the number of hidden nodes poses as a structural parameter that needs to be predefined, while parametric settings of the hidden nodes (for example, the impact factors of the RBF nodes, the biases and/or input weights of the additive nodes) are randomly assigned. 
Given training samples $\left\{\left(\mathbf{x}_{i}, \mathbf{t}_{i}\right) \mid \mathbf{x}_{i} \in \mathbf{R}^{d}, \mathbf{t}_{i} \in \mathbf{R}^{m}\right\}_{i=1}^{N}$, where $N$ is the number of instances, $d$ is the dimensions, $m$ is the number of output nodes. For regression problems $m=1$, while for classification problems $m$ is the number of categories, classes or labels. The output function of ELM for SLFNs is given by

$$
\boldsymbol{f}(\mathbf{x})=\sum_{i=1}^{L} \beta_{i} h\left(\mathbf{x}, \mathbf{a}_{i}, b_{i}\right)=\boldsymbol{h}(\mathbf{x}) \boldsymbol{\beta}
$$

where $L$ is the number of hidden nodes, $\boldsymbol{\beta}=\left[\beta_{i}, \ldots, \beta_{L}\right]^{T}$ is the vector of output weights, $\mathbf{a}_{i}$ is the center of RBF nodes or input weights of additive nodes, $b_{i}$ is the impact factor of RBF nodes or bias of additive nodes, and $h(\cdot)$ is the activation function which is but not limited to Sigmoid, Sine and hardlim functions. The ELM can be solved as a constrained optimization problem [14, 10, 15]:

$$
\operatorname{Minimize}_{\boldsymbol{\beta}}:\|\mathbf{H} \boldsymbol{\beta}-\mathbf{T}\|_{p}^{\alpha_{1}}+\frac{C}{2}\|\boldsymbol{\beta}\|_{q}^{\alpha_{2}}
$$

where $\alpha_{1}>0, \alpha_{2}>0, p, q=0, \frac{1}{2}, 1,2, \ldots, F,+\infty$ and $C$ is control parameter for a tradeoff between structural risk and empirical risk, $\mathbf{H}$ is the hidden-layer output matrix

$$
\mathbf{H}=\left[\begin{array}{ccc}
h\left(\mathbf{w}_{1}, \mathbf{x}_{1}, b_{1}\right) & \cdots & h\left(\mathbf{w}_{L}, \mathbf{x}_{1}, b_{L}\right) \\
\vdots & \ddots & \vdots \\
h\left(\mathbf{w}_{1}, \mathbf{x}_{N}, b_{1}\right) & \cdots & h\left(\mathbf{w}_{L}, \mathbf{x}_{N}, b_{L}\right)
\end{array}\right] \text { and } \mathbf{T}=\left[\begin{array}{c}
\mathbf{t}_{1}^{T} \\
\vdots \\
\mathbf{t}_{N}^{T}
\end{array}\right]
$$

Numerous efficient methods can be used to calculate the output weights $\boldsymbol{\beta}$ including but not limited to the orthogonal projection method, iterative methods [16], and eigenvalue decomposition method [17]. When $p, q=F$ and $\alpha_{1}, \alpha=2$, a popular and efficient closed-form solution [15] is:

$$
\boldsymbol{\beta}= \begin{cases}\mathbf{H}^{\top}\left(C \mathbf{I}+\mathbf{H} \mathbf{H}^{\top}\right)^{-1} \mathbf{T} & N \geq L \\ \left(C \mathbf{I}+\mathbf{H}^{\top} \mathbf{H}\right)^{-1} \mathbf{H}^{\top} \mathbf{T} & N \leq L\end{cases}
$$




\subsection{Kernel Extreme Learning Machine}

As proposed in [15], if $\boldsymbol{h}(\cdot)$ is unknown, i.e., an implicit function, one can apply the Mercer's conditions on ELM, and define a kernel matrix for ELM as follows:

$$
\mathbf{K}_{E L M}=\mathbf{H H}^{T}: \mathbf{K}_{E L M i, j}=\boldsymbol{h}\left(\mathbf{x}_{i}\right) \cdot \boldsymbol{h}\left(\mathbf{x}_{j}\right)=\kappa\left(\mathbf{x}_{i}, \mathbf{x}_{j}\right)
$$

Then, substituting (5) (4) into (1), we can obtain the kernel form of the output function as follows,

$$
\boldsymbol{f}(\mathbf{x})=\left[\begin{array}{c}
\kappa\left(\mathbf{x}, \mathbf{x}_{1}\right) \\
\vdots \\
\kappa\left(\mathbf{x}, \mathbf{x}_{N}\right)
\end{array}\right]^{T}\left(C \mathbf{I}+\mathbf{K}_{E L M}\right)^{-1} \mathbf{T}
$$

Similar to SVM [2] and LS-SVM [3], $\boldsymbol{h}(\mathbf{x})$ need not be known; instead, its kernel $\kappa(\mathbf{u}, \mathbf{v})$ (e.g., Gaussian kernel $\left.\kappa(\mathbf{u}, \mathbf{v})=\exp \left(\|\mathbf{u}-\mathbf{v}\|^{2} / \sigma\right)\right)$ can be provided. $L$ need not be available beforehand either. The experimental and theoretical analysis of [15] showed that KELM produces improved generalization performance over the SVM/LS-SVM. The work, however was established only on small datasets. When dealing with Big data, however, the training time of $\mathcal{O}\left(N^{3}\right)$ and kernel matrix size of $\mathcal{O}\left(N^{2}\right)$ becomes a significant concern [18].

\section{Fast Reduced Kernel Extreme Learning Machine}

For $N$ arbitrary distinct samples $\left\{\left(\mathbf{x}_{i}, \mathbf{t}_{i}\right) \mid \mathbf{x}_{i} \in \mathbf{R}^{d}, \mathbf{t}_{i} \in \mathbf{R}^{m}\right\}_{i=1}^{N}$, the inputs are denoted as $\mathbf{X}=\left\{\mathbf{x}_{i} \mid \mathbf{x}_{i} \in \mathbf{R}^{d}\right\}_{i=1}^{N}$ and the outputs are denoted as $\mathbf{T}=\left\{\mathbf{t}_{i} \mid \mathbf{t}_{i} \in\right.$ $\left.\mathbf{R}^{m}\right\}_{i=1}^{N}$. On regression problem, $\mathbf{t}_{i} \in \mathbb{R}^{m}$ is a continuous real vector. On $m$ categories multi-class classification problem, output $\mathbf{t}_{i} \in \mathbb{R}^{m}$ is a $m$-dimensional boolean vector $\{0,1\}^{m}$. If the class label is $p$, the expected output vector of the $m$ outputs is $\mathbf{t}_{i}=[0, \ldots, 0,1,0, \ldots, 0]_{1 \times m}$. In this case, only the $p$-th element of 
$\mathbf{t}_{i}=\left[t_{1}, t_{2}, \ldots, t_{m}\right]$ has value ' 1 ', while the rest of the elements are ' 0 '. Binary classification is considered as a special case of the multi-class classification problem where $m=2$. Differing from the conventional kernel-based algorithms (for example conventional SVM (LibSVM) [19] and BSGD-SVM [20]) which involves some iterative optimization scheme to identify the support vectors, or eventually using all the training sample as support vectors (for example, KELM and LS-SVM), we assert in this work that the support vectors can be randomly selected from the instances of the training data set. Since the current work is derived from KELM but only uses a reduced kernel matrix instead of the full kernel matrix to build the model, the proposed algorithm is labeled here as the Reduced Kernel Extreme Learning (RKELM). In what follows, we shall describe the proposed algorithm in details.

The SLFN with kernel function $\kappa(\cdot, \cdot)$ and $L$ support vectors $\mathbf{X}_{L}=\left\{\mathbf{x}_{i} \mid \mathbf{x}_{i} \in\right.$ $\left.\mathbf{R}^{d}\right\}_{i=1}^{L}$ can be mathematically modeled as,

$$
\sum_{s=1}^{L} \boldsymbol{\beta}_{s} \kappa\left(\mathbf{x}_{i}, \mathbf{x}_{s}\right)=\mathbf{t}_{i}, i=1,2, \ldots, N
$$

or compressed in the matrix form,

$$
\mathbf{K}_{N \times L} \boldsymbol{\beta}=\mathbf{T}
$$

where $\mathbf{K}_{N \times L}=\kappa\left(\mathbf{X}, \mathbf{X}_{L}\right)$ is the reduced kernel matrix, $\boldsymbol{\beta}=\left[\boldsymbol{\beta}_{1}, \boldsymbol{\beta}_{2}, \ldots, \boldsymbol{\beta}_{L}\right]$ is the output weight vector or matrix.

Theorem 1. For $N$ arbitrary distinct samples $\left\{\left(\mathbf{x}_{i}, \mathbf{t}_{i}\right) \mid \mathbf{x}_{i} \in \mathbf{R}^{d}, \mathbf{t}_{i} \in \mathbb{R}^{m}\right\}_{i=1}^{N}$, if a SLFN with SPD kernel and $L=N$ random support vectors $\mathbf{X}_{L}=R(\mathbf{X})$ is considered (i.e., all training samples are support vectors), then the kernel matrix $\mathbf{K}_{N \times N} \in \mathbf{R}^{N \times N}$ obtained from $N$ samples and $N$ support vectors is invertible and $\left\|\mathbf{K}_{N \times N} \boldsymbol{\beta}-\mathbf{T}\right\|^{2}=0$. (Proof is provided in Appendix A) 
Theorem 1 implies that, if $\kappa(\cdot, \cdot)$ is a strict positive definite (SPD) kernel [2], the SLFNs with $L=N$ random support vectors can learn $N$ distinct samples at zero error. This implies that $\left\|\mathbf{K}_{N \times N} \boldsymbol{\beta}-\mathbf{T}\right\|=0$. Furthermore, given any small positive value $\epsilon$ and $S P D$ kernel, there should exist $L \leq N$ random support vectors such that for $N$ arbitrary distinct samples $\left\{\left(\mathbf{x}_{i}, \mathbf{t}_{i}\right)\right\}_{i=1}^{N},\left\|\mathbf{K}_{N \times L} \boldsymbol{\beta}-\mathbf{T}\right\|<\epsilon$.

In many real world applications of the Big data era, one can safely assume that the number of support vectors $L$ shall always be less than $N$, and hence, the training error cannot be made exactly zero but approaches a non-zero training error $\epsilon$. Similar to Eq. (2), the RKELM with optimization constraints such as the $\ell_{2}$ norm minimization can be formulated as

$$
\begin{aligned}
& \text { Minimize } \frac{C}{2}\|\boldsymbol{\beta}\|^{2}+\frac{1}{2}\|\boldsymbol{\xi}\|^{2} \\
& \text { Subject to : } \boldsymbol{\xi}=\mathbf{K}_{N \times L} \boldsymbol{\beta}-\mathbf{T}
\end{aligned}
$$

Based on the KKT theorem, the solution is derived as

$$
\boldsymbol{\beta}=\left(C \mathbf{I}+\mathbf{K}_{N \times L}^{T} \mathbf{K}_{N \times L}\right)^{-1} \mathbf{K}_{N \times L}^{T} \mathbf{T}
$$

The proposed fast RKELM is summarized in Algorithm 1 .

Remark 1. Although the KELM does not involve an iterative learning procedure, the time complexity $\mathcal{O}\left(N^{3}\right)$ can still be very high, and often higher than many iterative-based algorithms. The core benefit of ELM, which is fast learning, is nonetheless lost. In contrast, the training cost of RKELM with $\mathcal{O}\left(L^{3}\right)$ is far lower than KELM with $\mathcal{O}\left(N^{3}\right)$, since $L \ll N$ in most cases. The space complexity on memory requirement of RKELM at $\mathcal{O}(N L)$ is also lower than that of KELM with $\mathcal{O}\left(N^{2}\right)$. Thus, the low computation cost of RKELM make it suitable for large scale data processing in the context of Big data. 


\section{Algorithm 1 Proposed Fast RKELM Algorithm Input:}

Given $N$ training samples $\aleph=\left\{\left(\mathbf{x}_{i}, \mathbf{t}_{i}\right) \mid \mathbf{x}_{i} \in \mathbf{R}^{d}, \mathbf{t}_{i} \in \mathbf{R}^{m}\right\}_{i=1}^{N}$, the number of support vectors $L$, and the kernel function $\kappa(\cdot, \cdot)$ such as Gaussian.

\section{Output:}

The output weights $\boldsymbol{\beta}$

1: Randomly select $L$ input samples from the training set as support vectors: $\mathbf{X}_{L} \leftarrow R(\mathbf{X})$

2: Generate the reduced kernel matrix from $N$ samples and $L$ support vectors: $\mathbf{K}_{N \times L}=\kappa\left(\mathbf{X}, \mathbf{X}_{L}\right)$

3: Estimate the output weights: $\boldsymbol{\beta}=\left(C \mathbf{I}+\mathbf{K}_{N \times L}^{T} \mathbf{K}_{N \times L}\right)^{-1} \mathbf{K}_{N \times L}^{T} \mathbf{T}$

Remark 2. It was proven in [12] that with sufficient hidden nodes, for a widespread forms of $h(\cdot)$, ELM can approximate any continuous target functions, that is,

$$
\lim _{L \rightarrow \infty}\left\|f_{L}(\mathbf{x})-f(\mathbf{x})\right\|=\lim _{L \rightarrow \infty}\left\|\sum_{i=1}^{L} \beta_{i} h_{i}(\mathbf{x})-f(\mathbf{x})\right\|=0
$$

However, when $h(\cdot)$ is a kernel, not all forms of kernel satisfy this condition. To date, it remains unestablished which forms of kernel possess universal learning properties. As one contribution of this paper, in Theorem 1 we prove that only the SPD kernels satisfy this condition.

Remark 3. Classification theorems in [12] [15] have shown that for given $m$ disjoint regions $K_{1}, K_{2}, \cdots, K_{m}$ in $\mathbf{R}^{d}$ and their corresponding labels $c_{1}, c_{2}, \cdots, c_{m}$, there exists a continuous function $f(\mathbf{x}) \in C\left(\mathbf{R}^{d}\right)$ or one compact set of $\mathbf{R}^{d}$ such that $f(\mathbf{x})=c_{i}$ if $\mathbf{x} \in K_{i}$. Theorem 1 indicates RKELM with SPD kernel can approximate any nonlinear function $f(\mathbf{x})$ only if $L=N$. Combining these two theorems, we confirm RKELM can separate these decision regions regardless of the shapes of the regions. 


\section{Related Work}

In this section, we discuss the relation of RKELM to other state-of-the-art algorithms including RSVM, KELM, RBF-network and the ELM-RBF.

\subsection{RSVM}

In RSVM [4], a small random sample of the dataset is used as representative samples of the original full dataset so as to accelerate optimization of the smooth support vector machine (SSVM) [4]. This is similar to the RKELM which uses the reduced kernel matrix to represent the full kernel matrix. Nevertheless, RSVM employs a globally quadratic convergent Newton algorithm in the training process, which involves solving a successive linear problem of the gradient of the objective function iteratively. RKELM on the other hand randomly selects the support vectors and computes the output weights without any iterative steps involved. Further, although RSVM can converge linearly to a solution, however, the universal approximation ability of RSVM remains unproven. In contrast, RKELM with SPD kernel is proven to possess universal approximation properties only if $L=N$.

\subsection{KELM}

From the equations $28 \mathrm{a}, 28 \mathrm{~b}$ and $28 \mathrm{c}$ in [15] we have,

$$
\boldsymbol{\beta}=\mathbf{H}^{T} \boldsymbol{\xi} / C
$$

Further, considering $\left\|\mathbf{H}^{T}\right\|^{2} \propto N L$ and $\|\boldsymbol{\xi}\|^{2} \propto N\|\overline{\boldsymbol{\xi}}\|^{2}$ where $\|\overline{\boldsymbol{\xi}}\|^{2}$ is the average of $\left\|\xi_{i}\right\|^{2}$, we have,

$$
\|\boldsymbol{\beta}\|^{2} \propto N^{2} L\|\overline{\boldsymbol{\xi}}\|^{2} / C
$$

Only focusing on $C$ and $L$, we have,

$$
\|\boldsymbol{\beta}\|^{2} \propto L / C
$$


It can be found that the task of $\|\boldsymbol{\beta}\|^{2}$ minimization can be optimized from two aspects: the number of hidden nodes $L$ and the control parameter $C$. In KELM [12], $L$ is fixed at $L=N$, so the minimization of $\|\boldsymbol{\beta}\|^{2}$ can only be achieved by regulating the parameter $C$. This limits the flexibility and controllability of the model. In contrast, since $L$ and $C$ are both flexible in RKELM, a more robust RKELM model can be attained, and in most cases RKELM is also more efficient than KELM since $L \ll N$.

\subsection{RBF Network and ELM-RBF}

The conventional RBF network (Lowe 1989) [21] focuses on specific RBF network with the same impact factor $\sigma$ assigned to all RBF hidden nodes: $\boldsymbol{f}(\mathrm{x})=$ $\sum_{i=1}^{L} \boldsymbol{\beta}_{i} h\left(\left\|\mathbf{x}-\mathbf{a}_{i}\right\| / \sigma\right)$, where the centers $\mathbf{a}_{i}$ and impact factor $\sigma$ of the RBF hidden nodes are typically determined based on the training data via some model selection scheme. In ELM-RBF, on the other hand, where $\boldsymbol{f}(\mathbf{x})=\sum_{i=1}^{L} \boldsymbol{\beta}_{i} h\left(\left\|\mathbf{x}-\mathbf{a}_{i}\right\| / \sigma_{i}\right)$, the RBF hidden nodes have different impact factors $\sigma_{i}$, and both $\mathbf{a}_{i}$ and $\sigma_{i}$ are random real values unrelated to the training data. In our proposed RKELM, where $\boldsymbol{f}(\mathbf{x})=\sum_{i=1}^{L} \boldsymbol{\beta}_{i} h\left(\left\|\mathbf{x}-\mathbf{x}_{i}\right\| / \sigma\right)$ or $\boldsymbol{f}(\mathbf{x})=\sum_{i=1}^{L} \boldsymbol{\beta}_{i} h\left(\left\|\mathbf{x}-\mathbf{x}_{i}\right\| / \sigma_{i}\right)$, the RBF hidden nodes can use the same or different impact factors; the centers $\mathrm{x}_{i}$ are randomly selected from the training data, and the $\sigma$ or $\sigma_{i}$ are random real values. For RKELM, we have the following theorems,

Theorem 2. Given $\kappa\left(\mathbf{x}, \mathbf{x}_{i}\right)=\exp \left(-\left\|\mathbf{x}-\mathbf{x}_{i}\right\|^{2} / \sigma\right)$ or $\kappa\left(\mathbf{x}, \mathbf{x}_{i}\right)=\exp (-\| \mathbf{x}-$ $\left.\mathbf{x}_{i} \|^{2} / \sigma_{i}\right)$, regardless of the impact factor, i.e., $\sigma$ is the same or $\sigma_{i}$ is different for each kernel, the generated kernel matrix has full rank. (Proof is provided in Appendix B)

Theorem 2 implies that RKELM possesses universal learning capability regardless of whether the impact factors are the same or different. J. Park [22] has shown that under certain mild conditions, conventional RBF-network [21] with the same 
impact factor possess universal approximation. Huang et al.[12] on the other hand showed that ELM-RBF possesses universal learning ability when the impact factors $\sigma_{i}$ are different and assigned with random real values.

Moreover, the nodes of RKELM can exist in the form of RBF kernel, Sigmoid kernel, wave kernel, etc. In contrast, those in the conventional RBF networks [21] only holds for specific type of RBF kernel.

\section{Experimental Study}

In this section, we verify the learning performance of the proposed RKELM by assessing it against several state-of-the-art machine learning algorithms including the conventional ELM, KELM, LS-SVM, conventional SVM, and RSVM using well established real world benchmark datasets in the field of regression, binary classification and multi-class classification. Note that the source codes of the stateof-the-art machine learning algorithms used in the present experimental study are based on those made available online at the websites of the respective researcher\$1. To maintain consistency and a fair comparison, our proposed RKELM ${ }^{2}$ is also

\footnotetext{
${ }^{1}$ The Matlab code of the conventional ELM and KELM are downloaded online from (http://www.ntu.edu.sg/home/egbhuang). The conventional SVM is based on the compiled C-coded SVM packages available in LIBSVM, i.e., (http://www.csie.ntu.edu.tw/ cjlin/libsvm/\#download). The LS-SVM Matlab code is obtained from (http://www.esat.kuleuven.be /stadius/lssvmlab/). The RSVM code is downloaded from http://dmlab8.csie.ntust.edu.tw/downloads/Download/SSVMtoolbox.zip. The LLSVM and BSGD code is obtained from (http://www.dabi.temple.edu /budgetedsvm/download.html). The LaSVM code is obtained from ( http://leon.bottou.org/projects/lasvm)

${ }^{2}$ The source code of RKELM is made available online at http: / / yunpan. cn/QDqESbIYiKhid
} 
implemented on MATLAB. All simulation runs made in the present study are conducted on a 2.6 GHZ CPU and 4G memory desktop PC, except for REAL-SIM, RCV1. BIN, RCV1. MUL and WEBSPAM which are conducted on a machine with 256G RAM.

\subsection{Data Sets}

In order to extensively validate the performances of the different algorithms considered, a wide range of datasets have been investigated in our experimental study, which comprises data of small (low) and big (high) instance size (dimensionality). The small-scale datasets include three multi-class classification datasets, four binary classification datasets and eight regression datasets as described in TABLE 1. The large-scale datasets includes three binary classification datasets and three multi-class classification datasets as described in TABLE 8 , Most of these datasets have been taken from the UCI Machine Learning Repository [23] , Statlib $]^{3}$ and LIBSVM website $]^{4}$. For each problem, the results reported are the average of 20 independent trials. All input attributes are normalized within the range of $[-1,1]$. The training and testing sets are defined as described in TABLE 1 and TABLE 8 , but the order of the dataset is randomly shuffled for each independent trial.

\subsection{User-Specified Parameters}

Unless explicitly stated, the Gaussian kernel $\kappa\left(\mathbf{x}, \mathbf{x}_{i}\right)=\exp \left(-\left\|\mathbf{x}-\mathbf{x}_{i}\right\|^{2} / \sigma\right)$ has been used in the experimental study of RKELM, KELM, LS-SVM, SVM and RSVM, while the sigmoid activation function $h(\mathbf{x}, \mathbf{a}, b)=1 /\left(1+e^{-\lambda(\mathbf{a} \cdot \mathbf{x}+b)}\right)$ has been considered in ELM. For RKELM and RSVM, the average results of 20 independent simulation trials on each combination of $(C, L)$ are then obtained and

\footnotetext{
${ }^{3} \mathrm{http} / / /$ lib.stat.cmu.edu/datasets/

${ }^{4}$ http://www.csie.ntu.edu.tw/ cjlin/libsvmtools/datasets/
} 
Table 1: Specification of small datasets

\begin{tabular}{|c|c|c|c|c|c|}
\hline & Dataset & \#Attributes & \#Classes & \#Train set & \#Test set \\
\hline \multirow{8}{*}{ 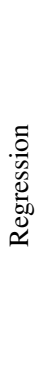 } & Auto-Mpg ${ }^{\mathrm{a}}$ & 7 & - & 320 & 72 \\
\hline & Abalone $^{a}$ & 8 & - & 3,000 & 1,177 \\
\hline & California Housing ${ }^{b}$ & 8 & - & 8,000 & 12,640 \\
\hline & YearPredictionMSD $^{a}$ & 90 & - & 463,715 & 51,630 \\
\hline & Breast cancer ${ }^{c}$ & 32 & - & 100 & 94 \\
\hline & Computer Activity ${ }^{c}$ & 21 & - & 4,000 & 4,192 \\
\hline & Triazines ${ }^{c}$ & 61 & - & 100 & 86 \\
\hline & Bank $^{c}$ & 9 & - & 4,500 & 3,692 \\
\hline \multirow{3}{*}{ 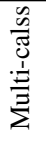 } & Image Segment $^{a}$ & 19 & 7 & 1,500 & 810 \\
\hline & Satellite Image ${ }^{a}$ & 36 & 6 & 4,435 & 2,000 \\
\hline & Shuttle $^{a}$ & 9 & 7 & 43,500 & 14,500 \\
\hline \multirow{4}{*}{ 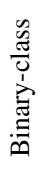 } & Wave form ${ }^{e f}$ & 21 & 2 & 4,000 & 1,000 \\
\hline & $\mathrm{USPS}^{e g}$ & 256 & 2 & 7,329 & 2,000 \\
\hline & Adult $^{e}$ & 122 & 2 & 32,562 & 16,282 \\
\hline & Skin Segment ${ }^{a}$ & 3 & 2 & 145,057 & 100,000 \\
\hline
\end{tabular}

${ }^{a}$ http://archive.ics.uci.edu/ml/datasets.

${ }^{\mathrm{b}}$ http://www.dcc.fc.up.pt/ ltorgo/Regression/cal_housing.html.

c http://www.cs.waikato.ac.nz/ml/weka/datasets.html.

${ }^{\mathrm{d}}$ http://www.csie.ntu.edu.tw/ cjlin/libsvmtools/datasets/multiclass.html.

e http://leon.bottou.org/papers/bordes-ertekin-weston-bottou-2005.

f Class 1 vs. rest.

g Class 0 vs. rest. 
Table 2: Parameters on Regression Applications

\begin{tabular}{lllllll}
\hline \multirow{2}{*}{ Dataset } & RKELM & ELM & KELM & LS-SVM & SVM & RSVM \\
\cline { 2 - 7 } & $(L, C, \sigma)$ & $(L, \lambda)$ & $(C, \sigma)$ & $(C, \sigma)$ & $(\# S V, C, \sigma)$ & $(\# S V, C, \sigma)$ \\
\hline Auto-Mpg & $\left(25,2^{-20}, 2^{2}\right)$ & $(25,1)$ & $\left(2^{-10}, 2^{2}\right)$ & $\left(2^{10}, 2^{2}\right)$ & $\left(168,2^{10}, 2^{-2}\right)$ & $\left(25,2^{20}, 2^{-2}\right)$ \\
Abalone & $\left(25,2^{-20}, 2^{2}\right)$ & $(25,1)$ & $\left(2^{-10}, 2^{2}\right)$ & $\left(2^{10}, 2^{2}\right)$ & $\left(1514,2^{10}, 2^{2}\right)$ & $\left(25,2^{20}, 2^{-2}\right)$ \\
California Housing & $\left(50,2^{-20}, 2^{0}\right)$ & $(50,1)$ & - & $\left(2^{10}, 2^{0}\right)$ & $\left(4122,2^{7}, 2^{0}\right)$ & $\left(50,2^{10}, 2^{0}\right)$ \\
YearPredictionMSD & $\left(30,2^{-20}, 2^{7}\right)$ & $(30,0.05)$ & - & - & $\left(4023,2^{5}, 2^{-5}\right)$ & $\left(30,2^{10}, 2^{-5}\right)$ \\
Breast cancer & $\left(20,2^{-10}, 2^{2}\right)$ & $(20,1)$ & $\left(2^{1}, 2^{2}\right)$ & $\left(2^{10}, 2^{-2}\right)$ & $\left(100,2^{10}, 2^{2}\right)$ & $\left(20,2^{10}, 2^{2}\right)$ \\
Computer activity & $\left(250,2^{-20}, 2^{0}\right)$ & $(250,1)$ & $\left(2^{10}, 2^{0}\right)$ & $\left(2^{10}, 2^{0}\right)$ & $\left(2083,2^{7}, 2^{0}\right)$ & $\left(250,2^{10}, 2^{0}\right)$ \\
Triazines & $\left(10,2^{-10}, 2^{5}\right)$ & $(10,1)$ & $\left(2^{3}, 2^{5}\right)$ & $\left(2^{4}, 2^{5}\right)$ & $\left(65,2^{2}, 2^{5}\right)$ & $\left(10,2^{10}, 2^{-5}\right)$ \\
Bank & $\left(200,2^{-20}, 2^{1}\right)$ & $(200,1)$ & $\left(2^{3}, 2^{1}\right)$ & $\left(2^{4}, 2^{1}\right)$ & $\left(2305,2^{2}, 2^{1}\right)$ & $\left(200,2^{20}, 2^{-1}\right)$ \\
\hline
\end{tabular}

the best average performance is reported. With KELM, LS-SVM and SVM, the best average performance of 20 trials on different $C=\left[2^{-30}, \cdots, 2^{5}\right]$ are reported. Last but not least, for ELM with Sigmoid function, the best average performances of 20 trials on each combination of $(\lambda, L)$ is then obtained and reported.

\subsection{Results on Small-Scale Regression Problems}

The performances of RKELM, ELM, KELM, LS-SVM and SVM are computed on eight real-world regression benchmark datasets that cover various fields of applications. The user-specified parameters considered in the simulation trials are then given in TABLE 2 TABLE 3 on the other hand tabulates the algorithmic performances obtained, including the training time and testing RMSE.

RKELM can be observed to incur the lowest training time efforts than the conventional ELM, KELM, LS-SVM, SVM and RSVM in most cases, while attaining competitive testing RMSE to the conventional ELM, LS-SVM, SVM and RSVM. Although the KELM is observed to report the lowest RMSE among all the algorithms considered, i.e., RKELM, ELM, SVM, LS-SVM and RSVM, it is shown to have incurred the highest training time. Taking the Computer activity dataset as an example, RKELM took 0.0355 seconds to train while the KELM incurred a CPU wall clock time of 13.946 seconds. Notably, RKELM is 300 times 
Table 3: Training time (in seconds) and testing error (in RMSE) on regression applications

\begin{tabular}{|c|c|c|c|c|c|c|c|c|c|c|c|c|}
\hline \multirow{2}{*}{ Dataset } & \multicolumn{2}{|c|}{ RKELM } & \multicolumn{2}{|c|}{ ELM } & \multicolumn{2}{|c|}{ KELM } & \multicolumn{2}{|c|}{ LS-SVM } & \multicolumn{2}{|c|}{ SVM } & \multicolumn{2}{|c|}{ RSVM } \\
\hline & $\mathrm{T}$ & & & Ts. & Tr.ti & $T_{0}$ & 11. & $F_{0} \mathrm{DN}$ & 11.UI & IS.KIV & 11. & $E$ \\
\hline Auto-Mpg & 0.0006 & 0.0737 & 0.0078 & 0.0778 & 0.0102 & 0.0718 & 0.0064 & 0.0720 & 0.0142 & 0.0763 & 0.0059 & 0.0828 \\
\hline balone & 0.0028 & 0.0765 & 0.0156 & 0.0782 & 4.6388 & 0.0750 & 1.2679 & 0.0754 & 0.668 & 0.0779 & 0.0111 & 0.0843 \\
\hline California & 0.0104 & 0.1290 & 0.0827 & 0.1320 & - & - & 15.898 & 0.1237 & 44.95 & 0.1196 & 0.1754 & 0.1373 \\
\hline MSD & 8.8039 & 0.1105 & 3.0228 & 0.1118 & - & - & - & - & 5.9372 & 0.1326 & 21.684 & 0.1170 \\
\hline Ditast & 0.0006 & 0.2794 & 0.0076 & 0.2 & 0.0013 & 3 & 0.0016 & 0.3047 & 5 & 0.2812 & 0.0056 & 02715 \\
\hline Computer & 0.0355 & 0.0295 & 0.0938 & 0.0308 & 13.946 & 0.0241 & 2.6813 & 0.0240 & 7.1277 & 0.0286 & 0.1323 & 0.0458 \\
\hline Triazines & 0.0005 & 0.1534 & 0.0259 & 0.1730 & 0.0024 & 0.1406 & 0.0037 & 0.1414 & 0.0031 & 0.1616 & 0.0141 & 0.1694 \\
\hline Bank & 0.0423 & 0.0435 & 0.1178 & 0.0430 & 19.321 & 0.0423 & 3.629 & 0.0426 & 2.0247 & 0.0433 & 0.1311 & 0.0475 \\
\hline
\end{tabular}

"_" : out of memory; Tr.time : Training Time; Ts.RMSE: Testing RMSE

faster than the KELM counterpart.

\subsection{Results on Small-Scale Classification Problems}

The RKELM performance has also been tested on seven small-scale classification problems. TABLE 4 summarizes the parameters settings, while TABLES 5,6 and 7 report the results of the respective algorithms. On multi-class classification problems, training time and testing accuracy is used to measure the performance of the algorithms. On binary classification problems, besides training time and testing accuracy, three additional performance metrics including Precision, Recall and F1-measure have been adopted. As observed from Tables 5, 6 and 7 , the training time taken by RKELM is much lower than ELM, KELM, LS-SVM, SVM and RSVM, and the testing accuracy is superior to ELM while competitive to LS-SVM/SVM/RSVM. Although RKELM did not perform better than KELM in terms of testing accuracy, it takes significantly smaller amount of time to train a model than the KELM. Taking the Satimage as an example, RKELM incurred a training time of 0.42 seconds, while the ELM at 1.53 seconds, KELM at 18.19 seconds, LS-SVM at 0.98 seconds, SVM at 1.317 seconds and the RSVM at 0.83 seconds. From these results, it is worth noting that RKELM is 150 times more ef- 
Table 4: Parameters on classification applications

\begin{tabular}{lllllll}
\hline \multirow{2}{*}{ Dataset } & RKELM & ELM & KELM & LS-SVM & SVM & RSVM \\
\cline { 2 - 6 } & $(L, C, \sigma)$ & $(L, \lambda)$ & $(C, \sigma)$ & $(C, \sigma)$ & $(\# S V, C, \sigma)$ & $(\# S V, C, \sigma)$ \\
\hline Segment & $\left(400,2^{25}, 2^{0}\right)$ & $\left(400,2^{-4}\right)$ & $\left(2^{5}, 2^{0}\right)$ & $\left(2^{10}, 2^{0}\right)$ & $\left(186,2^{5}, 2^{0}\right)$ & $\left(400,2^{10}, 2^{0}\right)$ \\
Satimage & $\left(400,2^{25}, 2^{1}\right)$ & $\left(500,2^{4}\right)$ & $\left(2^{5}, 2^{0}\right)$ & $\left(2^{10}, 2^{0}\right)$ & $\left(2012,2^{5}, 2^{1}\right)$ & $\left(400,2^{5}, 2^{-1}\right)$ \\
Shuttle & $\left(300,2^{20}, 2^{-3}\right)$ & $(300,1)$ & - & - & $\left(279,2^{10}, 2^{3}\right)$ & $\left(300,2^{10}, 2^{3}\right)$ \\
Skin & $\left(300,2^{20}, 2^{-3}\right)$ & $(300,1)$ & - & - & $\left(186,2^{10}, 2^{-3}\right)$ & $\left(500,2^{10}, 2^{-3}\right)$ \\
Waveform & $\left(250,2^{20}, 2^{1}\right)$ & $(250,1)$ & $\left(2^{3}, 2^{1}\right)$ & - & $\left(968,2^{1}, 2^{1}\right)$ & $\left(250,2^{10}, 2^{-1}\right)$ \\
USPS & $\left(500,2^{20}, 2^{8}\right)$ & $(500,1)$ & - & - & $\left(208,2^{5}, 2^{-8}\right)$ & $\left(500,2^{10}, 2^{-8}\right)$ \\
Adult & $\left(600,2^{20}, 2^{8}\right)$ & $(600,1)$ & - & - & $\left(11483,2^{5}, 2^{-8}\right)$ & $\left(600,2^{10}, 2^{-8}\right)$ \\
\hline
\end{tabular}

ficient compared to the KELM and 3 times faster than SVM. KELM and LS-SVM have been noted to reach the "run out of memory" state on a desktop computer that is equipped with a memory size of $4 \mathrm{G}$. The results also indicate that although KELM can produce the best generalization performance, the high time and memory complexities requirements of KELM makes it inappropriate for dealing with the many challenges of large-scale data, which is prevalent in the era of Big data.

On binary classification datasets, we can find that RKELM can obtain competitive or improved Precision, Recall and F1-measure results. Taking Waveform dataset as an example, in which the proportion of positive and negative samples is $N_{p} / N_{n}=0.6738$, RKELM can obtain the performance Precision=88.66\%, Recall $=87.14 \%$ and $\mathrm{F} 1=87.90 \%$, which is an improvement over the conventional ELM $(88.64 \%, 83.38 \%$ and $85.93 \%)$ on all three metrics, while competitive to KELM in terms of Precision (89.64\%) and an improvement over the KELM in Recall (82.20\%) and F1(85.76\%). 
Table 5: Performance comparison of RKELM, ELM, KELM, LS-SVM, SVM and RSVM on multi-class classification applications

\begin{tabular}{|c|c|c|c|c|c|c|c|c|c|c|c|c|}
\hline \multirow{2}{*}{ Dataset } & \multicolumn{2}{|c|}{ RKELM } & \multicolumn{2}{|c|}{ ELM } & \multicolumn{2}{|c|}{ KELM } & \multicolumn{2}{|c|}{ LS-SVM } & \multicolumn{2}{|c|}{ SVM } & \multicolumn{2}{|c|}{ RSVM } \\
\hline & Tr. time & Ts. rate & Tr. time & Ts. rate & Tr. time & e Ts. rate & Tr. time & Ts. rate & Tr. time & Ts. rate & Tr. time & Ts. rate \\
\hline Segment & 0.03 & $96.99 \%$ & 0.07 & $95.43 \%$ & 0.47 & $97.68 \%$ & 159.38 & $97.37 \%$ & 0.150 & $96.72 \%$ & 0.76 & $96.30 \%$ \\
\hline Satimage & 0.42 & $91.31 \%$ & 1.53 & $89.21 \%$ & 18.19 & $91.83 \%$ & 0.98 & $91.79 \%$ & 1.317 & $91.67 \%$ & 0.83 & $90.44 \%$ \\
\hline Shuttle & 0.67 & $99.66 \%$ & 0.76 & $99.36 \%$ & - & - & - & - & 1.55 & $\mathbf{9 9 . 9 2 \%}$ & 31.62 & $99.78 \%$ \\
\hline
\end{tabular}

"_, : out of memory, Tr.time : Training time (in seconds), Ts.rate: Testing accuracy

Table 6: Performance comparison of RKELM, Conventional ELM and KELM on binary classification applications

\begin{tabular}{|c|c|c|c|c|c|c|c|c|c|c|c|c|c|c|c|}
\hline \multirow{2}{*}{ Dataset } & \multicolumn{5}{|c|}{ RKELM } & \multicolumn{5}{|c|}{ ELM } & \multicolumn{5}{|c|}{ KELM } \\
\hline & Tr. time & Ts. rate & Precision & Recall & $\mathrm{F} 1$ & Tr. time & e Ts. rate & Precision & Recall & F1 & Tr. time & e Ts. rate & Precision & Recall & $\mathrm{F} 1$ \\
\hline Waveform & 0.04 & $91.48 \%$ & $88.66 \%$ & $87.14 \%$ & $87.90 \%$ & 0.09 & $91.24 \%$ & 6 $88.64 \%$ & $83.38 \%$ & $85.93 \%$ & to 15.36 & $91.14 \%$ & $89.64 \%$ & $82.20 \%$ & $85.76 \%$ \\
\hline USPS & 0.24 & $99.55 \%$ & $99.70 \%$ & $97.08 \%$ & $98.37 \%$ & 0.47 & $99.44 \%$ & $699.10 \%$ & $96.78 \%$ & $97.93 \%$ & $6 \quad 62.3$ & $99.49 \%$ & $98.26 \%$ & $98.83 \%$ & $98.54 \%$ \\
\hline Adult & 1.04 & $85.25 \%$ & $72.98 \%$ & $59.28 \%$ & $65.42 \%$ & 2.01 & $84.99 \%$ & $73.20 \%$ & $57.18 \%$ & $64.20 \%$ & $6 \quad-$ & - & - & - & - \\
\hline Skin & 2.85 & $99.84 \%$ & $96.89 \%$ & $99.99 \%$ & $98.35 \%$ & 3.28 & $99.3 \%$ & $96.77 \%$ & $99.88 \%$ & $98.24 \%$ & $6 \quad-$ & - & - & - & - \\
\hline
\end{tabular}

"_" : out of memory, Tr.t : Training Time (Seconds), Ts.acc: Testing Accuracy

Table 7: Performance comparison of RKELM, SVM and RSVM on binary classification applications

\begin{tabular}{|c|c|c|c|c|c|c|c|c|c|c|c|c|c|c|c|}
\hline \multirow{2}{*}{ Dataset } & \multicolumn{5}{|c|}{ RKELM } & \multicolumn{5}{|c|}{ SVM } & \multicolumn{5}{|c|}{ RSVM } \\
\hline & Tr. time & Ts. rate & Precision & Recall & $\mathrm{F} 1$ & Tr. time & e Ts. rate & Precision & Recall & $\mathrm{F} 1$ & Tr. time & Ts. rate & Precision & Recall & F1 \\
\hline Waveform & 0.04 & $91.48 \%$ & $88.66 \%$ & $87.14 \%$ & $87.90 \%$ & 0.337 & $90.92 \%$ & $85.71 \%$ & $88.12 \%$ & $86.90 \%$ & b $\quad 0.43$ & $90.32 \%$ & $83.38 \%$ & $84.69 \%$ & $84.03 \%$ \\
\hline USPS & 0.24 & $99.55 \%$ & $99.70 \%$ & $97.08 \%$ & $98.37 \%$ & 1.56 & $99.61 \%$ & $98.52 \%$ & $98.52 \%$ & $98.52 \%$ & b 1.29 & $99.34 \%$ & $97.35 \%$ & $97.93 \%$ & $97.64 \%$ \\
\hline Adult & 1.04 & $85.25 \%$ & $72.98 \%$ & $59.28 \%$ & $65.42 \%$ & 88.91 & $84.94 \%$ & $72.83 \%$ & $57.85 \%$ & $64.48 \%$ & b 6.93 & $85.11 \%$ & $72.80 \%$ & $59.02 \%$ & $65.19 \%$ \\
\hline Skin & 2.85 & $99.84 \%$ & $99.82 \%$ & $99.99 \%$ & $99.90 \%$ & 5.49 & $99.95 \%$ & $99.85 \%$ & $99.99 \%$ & $99.92 \%$ & o 79.50 & $99.74 \%$ & $96.89 \%$ & $99.19 \%$ & $98.02 \%$ \\
\hline
\end{tabular}

"_" : out of memory, Tr.time : Training Time (in seconds), Ts.rate: Testing accuracy 


\subsection{Results on Large Scale Problems}

TABLE 8 summarizes the large-scale data sets used in the experiments. The data sets are part of the LibSVM binary data collection 5 Besides ELM, we pit our method against other state-of-art large-scale algorithms including LLSVM [5], BSGD [20] and DTSVM [7]. TABLES 9 and 10 tabulates the experimental results of multi-class and binary classification applications, respectively. From the results in TABLES 9 and 10 , it can be observed that with respect to the ELM, RKELM exhibited improved generalization performances and incurred a lower training time on all six data sets considered. For example on REAL-SIM, RKELM took $29 \mathrm{sec}-$ onds to achieve the $95.89 \%$ test accuracy while ELM took 41 seconds to achieve an accuracy of $92.93 \%$; on the bigger WEBSPAM dataset, RKELM took 2,583 seconds to achieve the $98.23 \%$ test performance, while ELM took 2,698 seconds to arrive at $94.22 \%$. The reason for the higher efficiency of RKELM than ELM lies in the sparsity of the data sets, and the vector production occuring on few non-zero elements in the RKELM, thus leading to the significant cost saving. In contrast, for ELM, despite the sparse data, the random input weights of ELM are not sparse, thus the production operation needs to be carried out on every elements. With respect to the other algorithms, although RKELM did not emerge as superior in terms of prediction accuracy on the RCV1.BIN, REAL-SIM and WEBSPAM datasets, the testing accuracy of $96.85 \%$ on RCV1.BIN, $95.89 \%$ on REAL-SIM and $98.23 \%$ on WEBSPAM, is found to be highly competitive to the existing state-of-the-art algorithms. Notably, in terms of training time, it is significantly more efficient than all the algorithms considered in all cases. For example, on the WEBSPAM dataset which has more than 16 million features and 300,000 training samples, RKELM only took 2,583 seconds to attain the $98.23 \%$

\footnotetext{
${ }^{5}$ http://www.csie.ntu.edu.tw/ cjlin/libsvmtools/datasets/
} 


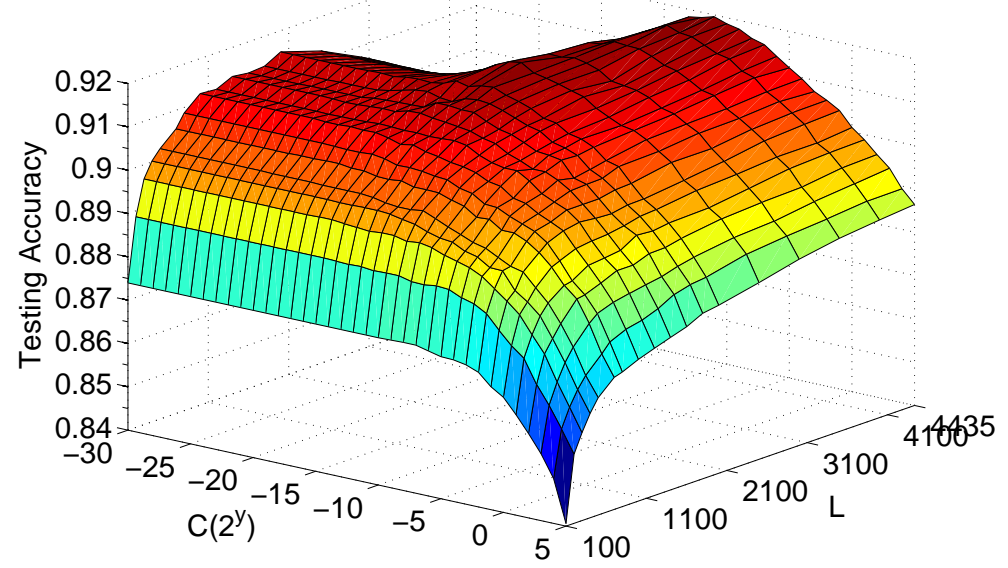

Figure 1: Testing accuracy change of RKELM on Satimage w.r.t $C$ and $L$

testing accuracy reported. Note that this represents a 24.3 times speed up over the DTSVM. To summarize, on big data problems where memory space and time complexity is a constant and concern, RKELM serves as an excellent choice of providing good balance in the training complexity and generalization accuracy.

\section{Parameters Sensitivity and Stability Analysis}

In this section, we presented a sensitivity study on the parameters of RKELM.

\subsection{Performances of RKELM w.r.t to Parameters $C$ and $L$}

Figure 1 summarizes the testing accuracy of RKELM on the Satimage w.r.t parameters $C$ and $L$. The detailed result is provided in Figure 2 to showcase the testing accuracies for different $C$ values, varying from $2^{-30}$ to $2^{5}$, under differing $L$ of $200,400,800,1200,1800,2500,4000$ and 4435 . From the figure, It can be observed that when $L$ is small (i.e., $L=200,400,800,1200,1800$ ), no form of over-fitting has been observed, hence indicating that any fine tuning of $C$ is ineffective for improving the testing accuracy. On the other hand, when $L$ becomes 
Table 8: Specification of Big Data sets

\begin{tabular}{lcccc}
\hline Dataset & \#Train set & \#Test set & \#Attributes & \#Classes \\
\hline REAL-SIM & 32,309 & 40,000 & 20,958 & 2 \\
RCV1.BIN & 677,399 & 20,242 & 47,236 & 2 \\
WEBSPAM & 300,000 & 50,000 & $16,609,143$ & 2 \\
RCV1.MUL & 518,571 & 15,564 & 47,236 & 53 \\
SECTOR & 6,412 & 3,207 & 55,197 & 105 \\
NEWS20.MUL & 15,935 & 3,993 & 62,061 & 20 \\
\hline
\end{tabular}

large (i.e., $L=2500,4000,4435$ ), the phenomenon of over-fitting can be observed. This indicates that a fine tuning of $C$ can be useful in mitigating the effects of over-fitting and improve generalization performance.
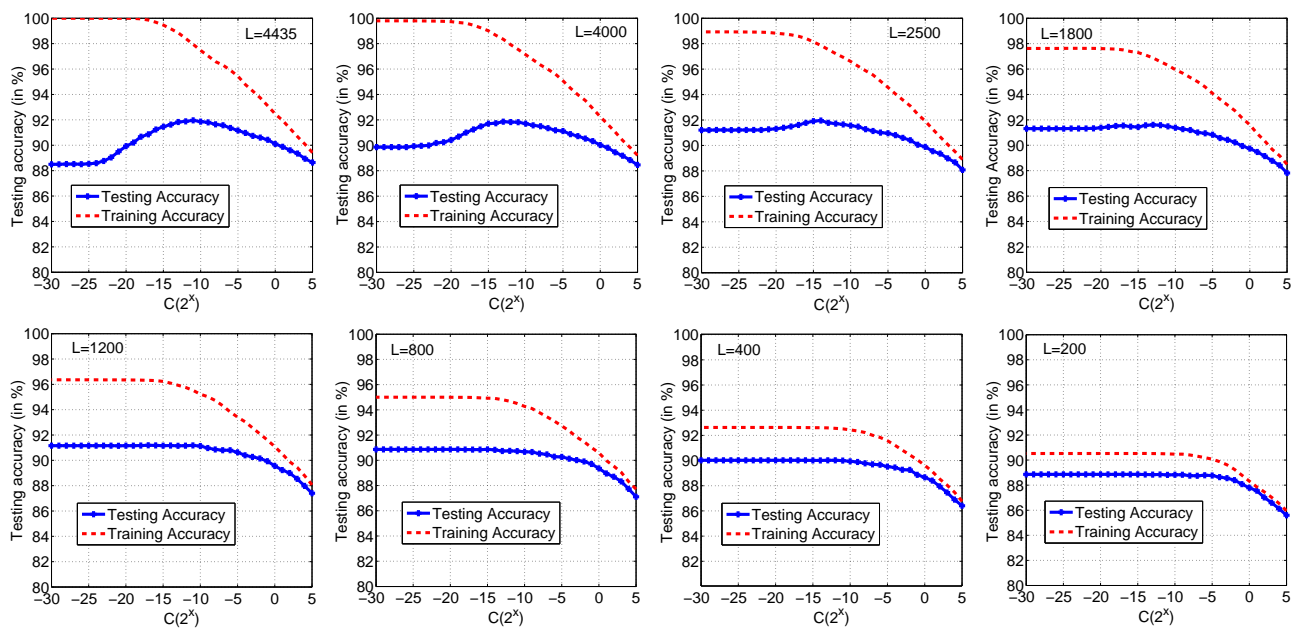

Figure 2: Performance accuracies of RKELM for different $L$ and $C$ values on Sat image dataset. $C$ is noted to pose a high influence on the performance of RKELM when the hidden nodes $L$ is large.

\subsection{Performances of RKELM for common $\sigma$ vs. different $\sigma_{i}$}

For RKELM with Gaussian kernel, the impact factor can be a common value $(\sigma)$ or different $\left(\sigma_{i}\right)$ for each kernel. Here, we study and analyze the effects of the impact factor on the performance of the RKELM as reported in TABLES 11 
Table 9: Performance comparison of RKELM to other state-of-the-art machine learning algorithms on large-scale multi-class classification applications

\begin{tabular}{cllll}
\hline \multicolumn{1}{c}{ Dataset } & Methods & Training time & Testing rate & Speedup \\
\hline \multirow{5}{*}{ NEWS20.MUL } & RKELM & $\mathbf{3 3 s}$ & $\mathbf{8 5 . 1 9 \%}$ & $1 \times$ \\
& ELM & $84 \mathrm{~s}$ & $82.46 \%$ & $1.4 \mathrm{x}$ \\
& LASVM [24] & $23,339 \mathrm{~s}$ & $83.10 \%$ & $12.8 \mathrm{x}$ \\
& DTSVM [24] & $3,053 \mathrm{~s}$ & $83.22 \%$ & $38.9 \mathrm{x}$ \\
& CBD & $39,590 \mathrm{~s}$ & $75.23 \%$ & $344 \mathrm{x}$ \\
\hline \multirow{5}{*}{ RCV1.MUL } & RKELM & $\mathbf{1 4 5 s}$ & $85.81 \%$ & $1 \times$ \\
& ELM & $521 \mathrm{~s}$ & $82.74 \%$ & $3.5 \times$ \\
& K-Pegasos [25] & N.A. & $84.50 \%$ & N.A. \\
& K-binaryLR [25] & N.A. & $83.00 \%$ & N.A. \\
& Multi-class LR [25] & N.A. & $\mathbf{8 8 . 5 0} \%$ & N.A. \\
\hline \multirow{5}{*}{ SECTOR } & RKELM & $\mathbf{1 8 s}$ & $\mathbf{9 3 . 8 6 \%}$ & $1 \times$ \\
& ELM & 20s & $91.22 \%$ & $1.04 \times$ \\
& LIBSVM(OVA) [7] & N.A. & $92.80 \%$ & $11.3 \times$ \\
& NB(OVA) [7] & N.A. & $64.30 \%$ & $24.3 \times$ \\
& LibSVM(BCH63) [7] & N.A. & $93.3 \%$ & $24.3 \times$ \\
& NB(BCH63) [7] & N.A. & $87.2 \%$ & $24.3 \times$ \\
\hline
\end{tabular}

Table 10: Performance comparison of RKELM to other state-of-the-art machine learning algorithms on large-scale binary classification applications

\begin{tabular}{clcccccl}
\hline Dataset & Methods & Training time & Testing rate & Precision & Recall & F1 & Speedup \\
\hline \multirow{5}{*}{ REAL-SIM } & RKELM & $\mathbf{2 9 s}$ & $95.89 \%$ & $94.49 \%$ & $88.66 \%$ & $91.48 \%$ & $1 \times$ \\
& ELM & $41 \mathrm{~s}$ & $92.93 \%$ & $89.14 \%$ & $83.37 \%$ & $86.16 \%$ & $1.4 \times$ \\
& TSVM [24] & $373 \mathrm{~s}$ & $93.10 \%$ & N.A. & N.A. & N.A. & $12.8 \times$ \\
& DA [24] & $1,129 \mathrm{~s}$ & $92.80 \%$ & N.A. & N.A. & N.A. & $38.9 \times$ \\
& RSVM & $10,002 \mathrm{~s}$ & $\mathbf{9 6 . 4 6 \%}$ & $94.54 \%$ & $88.72 \%$ & $91.53 \%$ & $344 \times$ \\
\hline \multirow{2}{*}{ RCV1.BIN } & RKELM & $\mathbf{4 0 6 s}$ & $96.85 \%$ & $96.40 \%$ & $97.89 \%$ & $97.14 \%$ & $1 \times$ \\
& ELM & $\mathbf{7 6 2 s}$ & $94.70 \%$ & $94.34 \%$ & $95.19 \%$ & $94.76 \%$ & $1.8 \times$ \\
& LLSVM & $1,800 \mathrm{~s}$ & $95.77 \%$ & $95.21 \%$ & $96.35 \%$ & $95.77 \%$ & $4.4 \times$ \\
& BSGD & $5,400 \mathrm{~s}$ & $97.08 \%$ & $96.63 \%$ & $97.91 \%$ & $97.26 \%$ & $13.3 \times$ \\
& SVM (RBF) & $68,687 \mathrm{~s}$ & $\mathbf{9 7 . 5 7 \%}$ & $96.84 \%$ & $98.11 \%$ & $97.47 \%$ & $169 \times$ \\
\hline \multirow{2}{*}{ WEBSPAM } & RKELM & $\mathbf{2 5 8 3 s}$ & $98.23 \%$ & $95.40 \%$ & $96.89 \%$ & $96.14 \%$ & $1 \times$ \\
& ELM & $2698 \mathrm{~s}$ & $94.22 \%$ & $91.11 \%$ & $92.81 \%$ & $91.95 \%$ & $1.04 \times$ \\
& CART [7] & $29,332 \mathrm{~s}$ & $98.44 \%$ & N.A. & N.A. & N.A. & $11.3 \times$ \\
& DTSVM [7] & $63,015 \mathrm{~s}$ & $\mathbf{9 9 . 0 3 \%}$ & N.A. & N.A. & N.A. & $24.3 \times$ \\
\hline
\end{tabular}


Table 11: Training time and testing RMSE of RKELM- $\sigma$, RKELM- $\sigma_{i}$ and ELM-RBF on regression problems

\begin{tabular}{lcccccc}
\hline \multirow{2}{*}{ Dataset } & \multicolumn{3}{c}{ RKELM- $\sigma^{*}$} & \multicolumn{2}{c}{ RKELM- $\sigma_{i}^{\dagger}$} & \multicolumn{2}{c}{ ELM-RBF } \\
\cline { 2 - 7 } & Train time & Test RMSE & Train time & Test RMSE & Train time & Test RMSE \\
\hline Auto & 0.0006 & $\mathbf{0 . 0 7 3 7}$ & $\mathbf{0 . 0 0 0 5}$ & 0.0757 & 0.0009 & 0.0756 \\
Abalone & $\mathbf{0 . 0 0 2 8}$ & $\mathbf{0 . 0 7 6 0}$ & 0.0029 & 0.0765 & 0.0046 & 0.0764 \\
California & $\mathbf{0 . 0 1 0 4}$ & $\mathbf{0 . 1 2 9 0}$ & 0.0123 & 0.1293 & 0.0155 & 0.1344 \\
YearPred & 8.8039 & 0.1105 & 8.7329 & $\mathbf{0 . 1 1 0 4}$ & $\mathbf{4 . 4 7 8 6}$ & 0.1138 \\
Breast & $\mathbf{0 . 0 0 0 6}$ & $\mathbf{0 . 2 7 9 4}$ & 0.0006 & 0.2812 & 0.0015 & 0.2853 \\
Computer & $\mathbf{0 . 0 3 5 5}$ & 0.0295 & 0.0424 & $\mathbf{0 . 0 2 4 8}$ & 0.0801 & 0.0315 \\
Triazines & 0.0005 & 0.1534 & $\mathbf{0 . 0 0 0 4}$ & $\mathbf{0 . 1 5 1 1}$ & 0.0004 & 0.1529 \\
Bank & 0.0423 & 0.0435 & $\mathbf{0 . 0 3 3 7}$ & 0.0431 & 0.0604 & $\mathbf{0 . 0 4 2 1}$ \\
\hline
\end{tabular}

RKELM- $\sigma^{*}:$ RKELM with same impact factor; RKELM- $\sigma_{i}^{\dagger}:$ RKELM with different impact factors

Table 12: Training time and testing classification accuracy of RKELM- $\sigma$, RKELM- $\sigma_{i}$ and ELMRBF on classification problems

\begin{tabular}{lcccccc}
\hline \multirow{2}{*}{ Dataset } & \multicolumn{2}{c}{ RKELM- $\sigma$} & \multicolumn{2}{c}{ RKELM- $\sigma_{i}$} & \multicolumn{2}{c}{ ELM-RBF } \\
\cline { 2 - 7 } & Train time & Test rate & Train time & Test rate & Train time & Test rate \\
\hline Segment & 0.0282 & $\mathbf{9 6 . 9 9 \%}$ & 0.0328 & $96.70 \%$ & 0.0982 & $95.17 \%$ \\
Satimage & 0.4210 & $91.31 \%$ & 0.4419 & $\mathbf{9 1 . 6 4 \%}$ & 1.9198 & $88.79 \%$ \\
Shuttle & 0.6699 & $99.66 \%$ & 0.6380 & $\mathbf{9 9 . 7 3 \%}$ & 0.9996 & $99.48 \%$ \\
Skin & 2.8506 & $99.85 \%$ & 2.1250 & $99.79 \%$ & 3.1019 & $99.41 \%$ \\
Waveform & 0.0405 & $\mathbf{9 1 . 4 8 \%}$ & 0.0465 & $91.28 \%$ & 0.0931 & $91.16 \%$ \\
USPS & 0.2401 & $99.55 \%$ & 0.2681 & $\mathbf{9 9 . 5 8 \%}$ & 0.5393 & $99.21 \%$ \\
Adult & 1.0411 & $85.25 \%$ & 1.1315 & $\mathbf{8 5 . 2 6 \%}$ & 2.0844 & $85.09 \%$ \\
\hline
\end{tabular}

and 12. The results obtained indicate that regardless of whether the impact factors of the kernels are the same or unique, RKELM remains capable of generating improved or competitive generalized performance to the ELM-RBF. For example, on the Sat image dataset, RKELM- $\sigma$ exhibited a testing accuracy of $91.31 \%$ and RKELM- $\sigma_{i}$ showcased a competitive testing accuracy of $91.64 \%$. Note that both RKELM- $\sigma_{i}$ and RKELM- $\sigma$ displayed improved generalized performance over the ELM-RBF (88.79\%). 


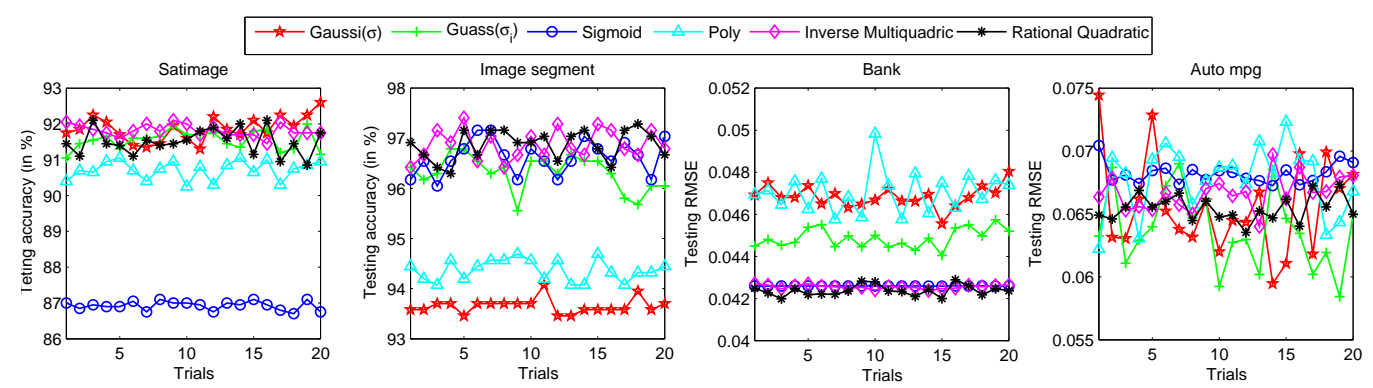

Figure 3: 20 trials testing accuracy of RKELM with different random support vectors

\subsection{Stability Analysis of the RKELM}

In the subsection, we further analyze the stability of the proposed RKELM experimentally. In particular, the average results of 20 independent simulation trials for different data subsets in each trial are plotted in Fig. 3. Due to space constraints, the results of two classification data sets (Satimage and Image segment) and two regression data sets (Bank and Auto Mpg) are presented as the representatives. Widely used kernel types including the Gaussian kernel, Sigmoid kernel, Polynomial kernel, Inverse Multiquadric kernel and Rational Quadratic kernel are considered here. From the results obtained, it can be observed that the testing accuracy performances do not vary much, regardless of the kernel function used.

For example, on the Satimage dataset, the standard derivation of RKELM$\sigma_{i}$ is about 0.01; on Auto Mpg, the standard derivation of RKELM- $\sigma_{i}$ is around 0.02. This indicates that there are no significant change in the testing accuracy across the 20 independent trials and stable generalization performance can be achieved with the use of random support vectors.

\subsection{The Influence of Data Density on RKELM Computational Efficiency}

In the conventional ELM, the random weights of ELM exists in the form of a dense matrix. In contrast to the conventional ELM, however, since the support 
vectors of RKELM are selected from the data itself, the sparse characteristics of the RKELM are naturally inherited from the sparsity of the dataset. In particular, since the input data and input weights are in the form of sparse matrices, the computational efficiency of RKELM is naturally higher than that of the ELM. To illustrate the impacts of data density on the computational efficiency of RKELM, three datasets of differing dimensionalities, i.e., $d=\left[10^{4}, 10^{5}, 10^{6}\right]$, have been generated here for experimental study. For each dataset, the degree of data density is designed to decrease according to the followings [0.6, 0.4, 0.2, 0.1, 0.08, 0.05, $0.02,0.01,0.005,0.002,0.001]$, and the training time of RKELM $(L=500)$ and ELM ( $L=500$ ) w.r.t different density level are then summarized in FIGURE4. It can be observed that the computational efficiency of RKELM is always higher than that of the conventional ELM. For data density in the range of $[0.2,0.01]$, the RKELM is noted to exhibit the largest improvement in computational efficiency over the ELM counterpart.
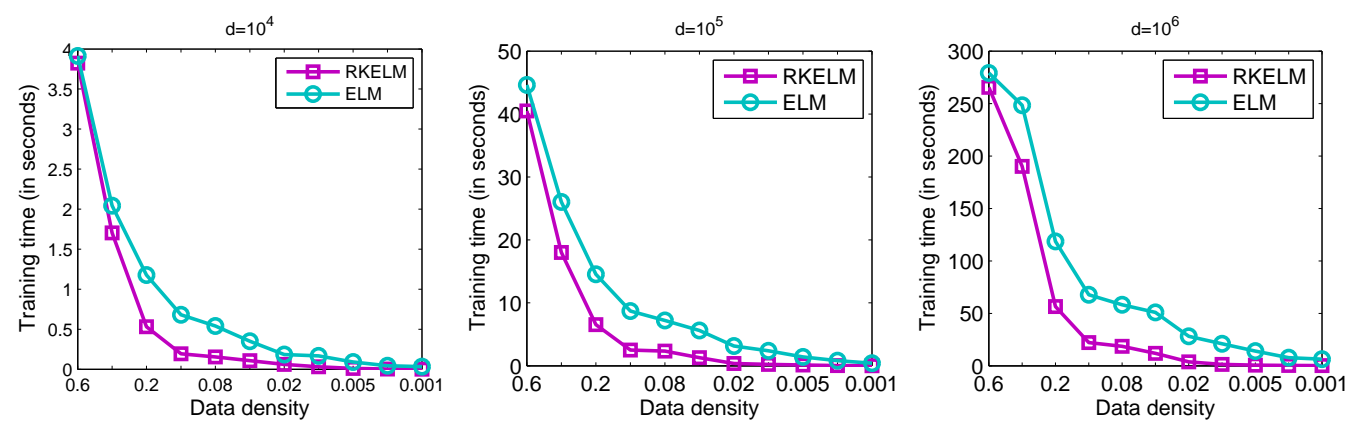

Figure 4: Training time comparison of RKELM and ELM with respect to data density

\section{Conclusion and Future Work}

Kernel-based algorithms including SVM, LS-SVM and KELM have been extremely popular in many real world applications involving classification and re- 
gression problems due to their excellent generalization performance reported. However, on Big data or large-scale problems, for conventional kernel-based approaches such as SVM, LS-SVM and KELM, the computational process of identifying support vectors can become very intensive due to the Big kernel matrix size, leading to poor computational efficiency. In this paper we proposed a fast kernel-based algorithm RKELM which randomly selects a subset of the available data as support vectors. Based on universal learning condition involving reduced kernel-based SLFN, we prove that RKELM can approximate any nonlinear functions with zero error only if the kernel is strictly positive definite. By avoiding iterative steps or the curse of kernel matrix size, significant cost savings in the RKELM training process can be readily attained. Widely experimental study on various of benchmarks shows that RKELM can produce competitive and stable performance at fast learning speed. However, the sparseness of RKELM is still an open problem and needs more study in the future.

\section{Acknowledgments}

This work is partially supported by the Computational Intelligence Graduate Laboratory at Nanyang Technological University. The first author is also grateful for the support from the Innovation fund research group 61221063; National Science Foundation of China 61100166, 61202184, 91118005, 91218301; Shaanxi New Star of Science \& Technology 2013 KJXX-29; New Star Team of Xian University of Posts \& Telecommunications; Provincial Key Disciplines Construction Fund of General Institutions of Higher Education in Shaanxi. 


\section{Appendices}

\section{A. Proof of Theorem 1}

From Theorem 3 we know, if kernel $\kappa(\cdot, \cdot)$ is $S P D$ kernel, then for every finite set $\left\{\mathbf{x}_{1}, \ldots, \mathbf{x}_{L}\right\} \subset \Omega$ where $\Omega$ is a domain (bounded or not) in $\mathbf{R}^{d}$, the kernel matrix $\mathbf{K}_{N \times N}$ has full rank, so $\mathbf{K}_{N \times N}$ is invertible and $\left\|\mathbf{K}_{N \times N} \boldsymbol{\beta}-\mathbf{T}\right\|=0$

\section{B. Proof of Theorem 2}

We first show the functions $\kappa\left(\cdot, \mathbf{x}_{i}\right)=\exp \left(-\left\|\mathbf{x}-\mathbf{x}_{i}\right\|^{2} / \sigma\right), i=1,2, \cdots, L$ are linear independent. The linear relation equation

$$
\sum_{i=1}^{L} \alpha_{i} \cdot \exp \left(-\left\|\mathbf{x}-\mathbf{x}_{i}\right\|^{2} / \sigma\right)=0, \alpha_{i} \in \mathbf{R}
$$

can be rewritten in the form,

$$
0=\sum_{i=1}^{L}\left(\alpha_{i} e^{-\left\|\mathbf{x}_{i}\right\|^{2} / \sigma}\right) e^{-\|\mathbf{x}\|^{2}} e^{2\left\langle\mathbf{x}, \mathbf{x}_{i}\right\rangle}=e^{-\|\mathbf{x}\|^{2}} \sum_{i=1}^{L} b_{i} e^{2\left\langle\mathbf{x}, \mathbf{x}_{i}\right\rangle}
$$

where $b_{i}=\alpha_{i} e^{-\left\|\mathbf{x}_{i}\right\|^{2} / \sigma}$, then we have,

$$
\sum_{i=1}^{L} b_{i} e^{2\left\langle\mathbf{x}, \mathbf{x}_{i}\right\rangle}=0
$$

Since this equation holds for any sample $\mathbf{x} \in \mathbf{R}^{d}$, one can get the homogenous system as following,

$$
\sum_{i=1}^{L} b_{i} e^{2\left\langle\mathbf{x}_{j}, \mathbf{x}_{i}\right\rangle}=0, \quad j=1,2, \cdots, L
$$

For a fixed sample $\mathbf{x} \in \mathbf{R}^{d}$, this is a Vandermonde system [26] of equations for the $b_{i}$. Consequently, $b_{i}=0$ and thus $\alpha_{i}=0$ for all $i$ as desired. So $\kappa\left(\cdot, \mathbf{x}_{i}\right)$ are linear independent. Further considering Theorem 3, we know Gaussian kernel matrix has full rank no matter whether the impact factor $\sigma$ is same or not in each kernel. 


\section{Theorem 3}

Theorem 3. If $\mathcal{H}$ is a reproducing kernel Hilbert function space with reproducing kernel $\kappa: \Omega \times \Omega \rightarrow \mathbf{R}$, then $\kappa$ is positive definite. Moreover, $\kappa$ is strictly positive definite if and only if $\kappa\left(\cdot, \mathbf{x}_{i}\right), i=1,2, \ldots, N_{\infty}$ are linearly independent where $N_{\infty}$ denotes one integer that may tend to be infinite.

Proof. For $N_{\infty}$ distinct samples $\mathbf{x}_{1}, \cdots, \mathbf{x}_{N_{\infty}}$ and nonzero vector $\mathbf{c} \in \mathbf{R}^{N_{\infty}}$ we have,

$$
\begin{aligned}
\sum_{j=1}^{N_{\infty}} \sum_{k=1}^{N_{\infty}} c_{j} c_{k} \kappa\left(\mathbf{x}_{j}, \mathbf{x}_{k}\right) & =\sum_{j=1}^{N_{\infty}} \sum_{k=1}^{N_{\infty}} c_{j} c_{k}<\kappa\left(\cdot, \mathbf{x}_{j}\right), \kappa\left(\cdot, \mathbf{x}_{k}\right)>_{\mathcal{H}} \\
& =<\sum_{j=1}^{N_{\infty}} c_{j} \kappa\left(\cdot, \mathbf{x}_{j}\right), \sum_{j=1}^{N_{\infty}} c_{j} \kappa\left(\cdot, \mathbf{x}_{j}\right)>_{\mathcal{H}} \\
& =\left\|\sum_{j=1}^{N_{\infty}} c_{j} \kappa\left(\cdot, \mathbf{x}_{j}\right)\right\|_{\mathcal{H}}^{2} \geq 0
\end{aligned}
$$

$\kappa$ is positive definite. Moreover, if $\kappa$ is strictly positive definite, then $\left\|\sum_{j=1}^{N_{\infty}} c_{j} \kappa\left(\cdot, \mathbf{x}_{j}\right)\right\|_{\mathcal{H}}^{2}>$ 0 . This means that for nonzero vector $\mathbf{c} \in \mathbf{R}^{N_{\infty}}, \sum_{j=1}^{N_{\infty}} c_{j} \kappa\left(\cdot, \mathbf{x}_{j}\right) \neq 0$, i.e., $\kappa\left(\cdot, \mathbf{x}_{j}\right)$ are linear independent. Conversely, if $\kappa\left(\cdot, \mathbf{x}_{j}\right)$ are linear independent, then for nonzero vector $\mathbf{c} \in \mathbf{R}^{N}, \sum_{j=1}^{N_{\infty}} c_{j} \kappa\left(\cdot, \mathbf{x}_{j}\right) \neq 0$ and thus $\sum_{j=1}^{N_{\infty}} \sum_{k=1}^{N_{\infty}} c_{j} c_{k} \kappa\left(\mathbf{x}_{j}, \mathbf{x}_{k}\right)>$ 0 , i.e. $\kappa$ is strictly positive definite. This means that for every finite set $\left\{\mathbf{x}_{1}, \ldots, \mathbf{x}_{N}\right\} \subset$ $\Omega$, if $\kappa$ is strictly positive definite, then the kernel matrix $\kappa\left(\mathbf{x}_{i}, \mathbf{x}_{j}\right)$ has full rank.

\section{References}

[1] P. Neuvial, Asymptotic results on adaptive false discovery rate controlling procedures based on kernel estimators, The Journal of Machine Learning Research 14 (1) (2013) 1423-1459.

[2] V. N. Vapnik, The Nature of Statistical Learning Theory, Springer-Verlag New York, Inc., New York, NY, USA, 1995. 
[3] J. A. K. Suykens, J. Vandewalle, Least squares support vector machine classifiers, Neural Process. Lett. 9 (3) (1999) 293-300.

[4] Y.-J. Lee, S.-Y. Huang, Reduced support vector machines: A statistical theory, IEEE Transactions on Neural Networks 18 (1) (2007) 1-13.

[5] K. Zhang, L. Lan, Z. Wang, F. Moerchen, Scaling up kernel SVM on limited resources: A low-rank linearization approach, 2012, pp. 1425-1434.

[6] Z. Wang, K. Crammer, S. Vucetic, Breaking the curse of kernelization: Budgeted stochastic gradient descent for large-scale svm training, J. Mach. Learn. Res. 13 (1) (2012) 3103-3131.

[7] F. Chang, C.-Y. Guo, X.-R. Lin, C.-J. Lu, Tree decomposition for large-scale SVM problems, J. Mach. Learn. Res. 11 (2010) 2935-2972.

[8] A. Bordes, S. Ertekin, J. Weston, L. Bottou, Fast kernel classifiers with online and active learning, J. Mach. Learn. Res. 6 (2005) 1579-1619.

[9] I. W. Tsang, J. T. Kwok, P.-M. Cheung, Core vector machines: Fast svm training on very large data sets, J. Mach. Learn. Res. 6 (2005) 363-392.

[10] G.-B. Huang, Q.-Y. Zhu, C.-K. Siew, Extreme learning machine: Theory and applications, Neurocomputing 70 (1) (2006) 489-501.

[11] G.-B. Huang, Q.-Y. Zhu, C.-K. Siew, Extreme learning machine: a new learning scheme of feedforward neural networks, in: 2004 IEEE International Joint Conference on Neural Networks, 2004. Proceedings, Vol. 2, 2004, pp. 985-990 vol.2.

[12] G.-b. Huang, L. Chen, C.-K. Siew, Universal approximation using incremental constructive feedforward networks with random hidden nodes, IEEE Transactions on Neural Networks 17 (4) (2006) 879-892. 
[13] G.-B. Huang, L. Chen, Convex incremental extreme learning machine, Neurocomputing 70 (168) (2007) 3056-3062.

[14] G.-B. Huang, An insight into extreme learning machines: Random neurons, random features and kernels, Cognitive Computation 6 (3) (2014) 376-390.

[15] G.-B. Huang, H. Zhou, X. Ding, R. Zhang, Extreme learning machine for regression and multiclass classification, IEEE Transactions on Systems, Man, and Cybernetics, Part B: Cybernetics 42 (2) (2012) 513-529.

[16] J. Luo, C.-M. Vong, P.-K. Wong, Sparse bayesian extreme learning machine for multi-classification, Neural Networks and Learning Systems, IEEE Transactions on 25 (4) (2014) 836-843.

[17] G. H. Golub, C. F. Van Loan, Matrix Computations (3rd Ed.), Johns Hopkins University Press, Baltimore, MD, USA, 1996.

[18] Y. Zhai, Y.-S. Ong, I. Tsang, The emerging "big dimensionality", IEEE Computational Intelligence Magazine 9 (3) (2014) 14-26.

[19] C.-C. Chang, C.-J. Lin, LIBSVM: A library for support vector machines, ACM Trans. Intell. Syst. Technol. 2 (3) (2011) 27:1-27:27.

[20] Z. Wang, K. Crammer, S. Vucetic, Breaking the curse of kernelization: Budgeted stochastic gradient descent for large-scale SVM training, J. Mach. Learn. Res. 13 (1) (2012) 3103-3131.

[21] D. Lowe, Adaptive radial basis function nonlinearities, and the problem of generalisation, in: Artificial Neural Networks, 1989., First IEE International Conference on (Conf. Publ. No. 313), 1989, pp. 171-175.

[22] J. Park, I. W. Sandberg, Universal approximation using radial-basis-function networks, Neural Comput. 3 (2) (1991) 246-257. 
[23] K. Bache, M. Lichman, UCI machine learning repository (2013). URL http: / / archive.ics.uci.edu/ml

[24] V. Sindhwani, S. S. Keerthi, Large scale semi-supervised linear SVMs, in: Proceedings of the 29th Annual International ACM SIGIR Conference on Research and Development in Information Retrieval, SIGIR '06, ACM, New York, NY, USA, 2006, pp. 477-484.

[25] D. Chen, W. Chen, Q. Yang, Characterizing inverse time dependency in multi-class learning, in: 2011 IEEE 11th International Conference on Data Mining (ICDM), 2011, pp. 1020-1025.

[26] C. Demeure, L. Scharf, Fast least squares solution of vandermonde systems of equations, in: Acoustics, Speech, and Signal Processing, 1989. ICASSP89., 1989 International Conference on, 1989, pp. 2198-2210 vol.4. doi: $10.1109 /$ ICASSP.1989.266900. 